\title{
Assessing the stability of long-horizon SSA forecasting
}

\author{
AndRey PePElyshev AND ANATOly ZhigluaVsKY*
}

We start with formalizing the problem of assessing the stability of long-horizon forecasting of a given family of forecasting techniques. Next, we argue that in some applications the so-called singular-spectrum analysis (SSA) could be a suitable family of techniques to consider. We then apply the proposed methodology to two simulated time series and also to the Earth temperature records. We demonstrate that the SSA forecasts of the temperatures appear to be more stable at present than three-four years ago.

AMS 2000 subJeCt Classifications: Primary 62M20. KeYwords AND PHRASES: Singular Spectrum Analysis, stability of forecasts, long-horizon forecasting, retrospective forecasts, SSA vector forecasting.

\section{ASSESSING THE STABILITY OF FORECASTS}

Assume that we have a time series $x_{1}, \ldots, x_{T}$ and our aim is to make an $h$-step forecast for this series, where the horizon $h$ is relatively large (for example, $h=100$ ). We assume that the structure of the series $x_{1}, x_{2}, \ldots$ is complex and may not be very stable; an implication of this is the inadequacy of the assumption of the stationarity of the series. We, however, assume that the changes in the structure do not occur often and these changes are relatively small. In particular, we assume that these changes are not going to dramatically alter the behaviour of the series during the forecasting period. If this assumption holds, then the past reflects the future and therefore forecasting makes sense.

We also assume that we have a family of forecasting techniques and wish to assess the reliability of the corresponding $h$-step forecasts. More precisely, we want to make an opinion about how much can we trust the $h$-step forecast $x_{T+h}$.

\section{Evaluation of the quality of forecasts}

To evaluate the accuracy and reliability of the forecasts, one can use a suitable combination of the following three approaches: (a) construction of confidence intervals; (b) assessment of retrospective forecasts; (c) checking the stability of forecasts.

\footnotetext{
${ }^{*}$ Corresponding author.
}

The approach (a) is based on the use of parametric models of the series and/or on the bootstrap techniques. These parametric models are either assumed (for example, ARIMA) or built in the process of analysis (SSA). In the long-horizon forecasting the use of confidence bounds is very limited and often even useless. For example, in the ARIMAtype models the confidence intervals become very wide for large $h$. In SSA and similar techniques the confidence intervals do not increase fast but they depend on the accuracy of the constructed model and on the fact that this model is not going to break during the forecasting period. Note that the SSA confidence intervals are shorter than the ARIMA confidence intervals even if the time series is an ARIMA process, see [3].

Retrospective forecasts (the approach (b)) are performed by truncating the series and forecasting values at the points temporarily removed. These forecasts can then be compared with the observed values of the time series for making an assessment of the quality of the forecasts. The use of the retrospective forecasts is important, especially when $h$ is small. In the situation we consider, its usefulness is very limited in view of the magnitude of $h$ and the instability of the structure of the series.

Despite the situation, we do not dismiss the approaches (a) and (b), in the present paper. We only concentrate on the approach (c); that is, on the assessing the stability of forecasts.

\section{Creation of the samples of the forecast values}

Assume that we have a family of forecasting techniques which is parameterized by a parameter $\theta \in \Theta \subset \mathbb{R}^{m}$. The set $\Theta$ is an $m$-dimensional set of admissible values; some components of $\theta \in \Theta$ may take values on the ordinal or even nominal scale (which would correspond to switches between groups of methods). Any $\theta \in \Theta$ defines a particular forecasting technique from the chosen family of techniques.

We may be interested in the forecast to horizons which are approximately $h$ rather than exactly $h$; that is, to the domain of horizons $g \in\left[h_{1}, h_{2}\right]$ with $0<h_{1} \leq h \leq h_{2}<\infty$. Choosing an interval of horizons rather than a single horizon makes sense, for example, if we are not interested in the quality of extracted seasonality components but are much more interested in the general tendency and the related variability of the forecasts. On the other hand, if we expect a strong trend and/or strong seasonality in the future behaviour of 
the series then the choice $h_{1} \cong h_{2}$ seems to be more reasonable.

For each time moment $t \leq T$, any $g>0$ and any $\theta \in \Theta$ we can build a $g$-step ahead forecast $\hat{x}_{t+g}(\theta)$ based on the information $x_{1}, \ldots, x_{t}$. Hence for any $t \leq T$ we may compute the following set of forecasting results:

$$
F_{t}=\left\{\hat{x}_{T+g}(\theta): g \in\left[h_{1}, h_{2}\right], \theta \in \Theta\right\} .
$$

In (1) the subscript $t$ designates that the forecasts are based on the use of the subseries $x_{1}, \ldots, x_{t}$.

If the main interest of the study is the quality of the $h$ step ahead forecasting procedures (for example, when we compare two families of forecasting techniques), then the following set of forecasting results may also be of interest: $\left\{\hat{x}_{t+g}(\theta): g \in\left[h_{1}, h_{2}\right], \theta \in \Theta\right\}$.

If $\Theta$ is a finite set then the sets $F_{t}$ are finite, otherwise (if some components of $\theta \in \Theta$ take values at an infinite set) we need to take a sufficiently representative sample of values $\theta_{i} \in \Theta$ and approximate the sets (1) by finite sets which we shall call samples (of the values of the forecasts). In what follows we assume that the set $\Theta=\left\{\theta_{1} \ldots, \theta_{m}\right\}$ is finite. The number of elements in the samples $F_{t}$ is $M=m\left(h_{2}-h_{1}+1\right)$.

Summarizing, our forecasting procedure allows us to get $T-T_{0}+1$ samples $F_{t}=\left\{f_{1}^{(t)}, \ldots, f_{M}^{(t)}\right\}$ computed according to (1) at all $t=T_{0}, \ldots, T$ where $T_{0}$ is the first time moment we applied the forecasting procedures.

\section{Comparison of the samples $F_{t}$}

We now need to compare the samples $F_{t}\left(t=T_{0}, \ldots, T\right)$ to evaluate the stability of the corresponding forecasts and decide whether at $t=T$ we have reached an acceptable level of stability.

The mean values of the samples $F_{t}$ are $\bar{f}_{t}=$ $\left(f_{1}^{(t)}+\cdots+f_{M}^{(t)}\right) / M$. Ideally, the sequence of these mean values should approach the true value of the expectation of $x_{T+h}$ under the following assumptions: $h_{1}=h_{2}, m \rightarrow \infty$, the model of the time series is signal + noise, the signal has been fully extracted and extrapolated by the forecasting techniques, and the structure of the series has stayed unchanged for a period of time significantly longer than $h$. These assumptions are unrealistic and are impossible to check (unless we are doing simulation experiments). Moreover, the fact whether the values $\bar{f}_{t}$ are approaching $\mathbf{E} x_{T+h}$ or not is not important if we consider the stability of the forecasts.

As measures of stability, we must consider the behaviour of some characteristics of variability of the samples $F_{t}$. The simplest and perhaps most natural among these characteristics is the (empirical) standard deviation of $F_{t}$ :

$$
s_{t}=\sqrt{\frac{1}{M-1} \sum_{i=1}^{M}\left(f_{i}^{(t)}-\bar{f}_{t}\right)^{2}} .
$$

We may say, for example, that the series $x_{1}, \ldots, x_{T}$ leads to a more stable forecast than the subseries $x_{1}, \ldots, x_{t}$ (with $t<T$ ) if the standard deviation $s_{T}$ is significantly smaller than the standard deviation $s_{t}$.

One of the reasons for the importance of $s_{t}$ is the observation that under some natural assumptions the lengths of the asymptotic confidence intervals for the mean values of the forecasts are proportional to the values of $s_{t}$. Note that we can also use some robust estimators of the standard deviation of the distribution which the sample $F_{t}$ corresponds to.

In addition to the standard deviation $s_{t}$, we would recommend to consider another important characteristic of sample variability, the range of the sample $F_{t}$ :

$$
R_{t}=\max _{i=1, \ldots, M} f_{i}^{(t)}-\min _{i=1, \ldots, M} f_{i}^{(t)}
$$

One may decide to prefer the range $R_{t}$ over the standard deviation $s_{t}$ if the main emphasis in the study is on the worst-case (guaranteed) performance of the forecasts rather than on their behaviour in most cases.

\section{SSA AND OTHER FAMILIES OF FORECASTING TECHNIQUES}

Combined error of any forecast is due to the random error (associated with noise) and the bias (associated with the chosen forecasting technique).

In this study, we treat the time series purely as a set of numbers. Usually, however, there is some randomness in the data which gives rise to some random error in the forecast, even when we try to filter the noise out (as SSA does). There is also bias in the forecast associated with the chosen model and the group of forecasting techniques: precise prediction of the future is not generally possible.

Thus, there is very little hope of building a very accurate forecast for the medium/long horizons. We however may hope to find a rich family of forecasting techniques whose forecasts are becoming more and more stable as the amount of information about the series increases.

\section{ARIMA and similar models}

The majority of the classical forecasting techniques (with ARMA as the main example) use the assumption of stationarity of the series; to achieve the stationarity it is customary to make the differencing of the series (leading to ARIMAtype models) or extract a non-stationary trend using regression. In addition to the assumption of stationarity, which we are not prepared to assume, there are at least two more obstacles preventing the use of ARIMA-type techniques in our studies: (a) the confidence intervals for the forecasted values at long horizons are typically very wide, and (b) the choice of parameters in these techniques is usually automatic and it is not obvious how to create a rich and representative family of these techniques. 
The use of change-point detection techniques

Since we assume that certain changes in the structure of the series $x_{1}, x_{2}, \ldots$ may occur, the following strategy for analyzing these series seems to be very reasonable: (a) use a suitable change-point detection technique, (b) if a change in the structure of the series is detected then disregard the initial part of the series, and (c) apply one of standard techniques for analyzing and forecasting the remaining part of the series.

However, we are not going to use this strategy. One of the reasons for this is that the use of change-point detection techniques would contradict to the main aim of the study which is the verification of the fact that all of the chosen past of the series is homogeneous enough to provide stable forecasts based on all previous information; a removal of a part of the series would confront this.

\section{Singular spectrum analysis (SSA)}

While using SSA neither a parametric model nor stationarity-type conditions have to be assumed for the time series; this makes SSA a model-free technique. SSA is robust to small changes in the structure of the series which makes SSA an ideal tool in our study. We shall be using the basic version described in the introduction to this volume [2]; note that we shall also be using the notation introduced in [2].

\section{SSA forecasting}

There are several ways of constructing forecasts based on the SSA decomposition of the series, see Chapter 2 in [3]. The most obvious way is to use the linear recurrent formula which the series reconstructed from $\tilde{\mathbf{X}}$ satisfies. We however prefer to use the so-called 'SSA vector forecast' ([3], Sect. 2.3.1). The main idea of the SSA vector forecasting algorithm is as follows. Selection of $r$ eigenvectors of $\mathbf{X X}^{T}$ leads to the creation of the subspace $S_{r}$. SVD properties provide us a hope that the $L$-dimensional vectors $\left\{X_{1}, \ldots, X_{K}\right\}$ lie close to this subspace. The forecasting algorithm then sequentially constructs the vectors $\left\{X_{K+1}, X_{K+2}, \ldots\right\}$ so that they stay as close as possible to the chosen subspace $S_{r}$.

\section{Choice of SSA parameters}

In the examples below, the length of the series is $T \cong 300$ and the forecasting horizon is $h \cong 100$. If the structure of the series is assumed stable then large values of $L$, of the order $L \cong 100$, should be preferred to small values, of the order $L \cong 10$. We, however, assume that the structure of the series is not rigid (despite in Example 2 where the structure is stable). In this case, large values of $L$ would not give SSA enough flexibility to react to the changes (which we assume rare and slow). Conversely, for small values of $L$, SSA may be too sensitive to the noise and small variations in the trend. It is therefore natural to select values of $L$ somewhere inbetween. Our choice is $20 \leq L \leq 50$ which we believe is a rather broad range.
The second SSA parameter to choose is $r$, the dimension of the subspace $S_{r}$. The choice of $r$ should depend on what do we intend to forecast. For example, if we observe some seasonal variations in the data and we want to forecast these variations, then we have to choose $r$ large enough to capture these variations (see Example 2 below). Alternatively, if the analysis shows that these variations are insignificant and the only tendency of interest is the general trend, then the value of $r$ should be small (see Example 1). There are several procedures (see e.g. [3]) for choosing the most suitable value of $r$ (roughly speaking, $r$ should be the smallest among those values of $r$ for which the residuals after signal extraction pass the chosen statistical tests for being a noise). These procedures, however, are often not very reliable and are not well suited for the long-term forecasting which is the purpose of our study.

We realize that whatever the rule of selection of $r$, some values of $r$ are too small, which leads to us missing parts of the signal, but other values of $r$ are too large, which means that we include a significant part of the noise into the 'reconstructed signal'. This, however, goes in line with the purpose of our study which is checking the stability of the forecasts with respect to both the signal and noise behavior.

\section{SIMULATED EXAMPLES}

In the following two examples we try to emulate some features of the real-life series considered in Section 4. In both examples, the length of the series is $T=300$ and the first time moment where the forecasts start is $N-n+1=230$. All forecasts are made by the SSA vector forecasting algorithm for $h$ steps, where either $h=100$ or $h \simeq 100$. The window length $L$ in the SSA algorithm takes all the values in the interval $L \in[20,50]$.

\section{Simulated example 1}

Let the time series be

$$
x_{t}= \begin{cases}-5(1-t / 200)^{2}+\varepsilon_{t} & \text { for } t=1, \ldots, 200 \\ \varepsilon_{t} & \text { for } t=201, \ldots, 300\end{cases}
$$

where $\varepsilon_{t}$ are independent Gaussian random variables with mean 0 and variance 1 . Note that there is a gradual change of structure of this series just before the time $t=200$.

We only consider a single realization of this series shown in Figure 1. This figure also shows a typical SSA approximation (which we obtain for $L=50$ and $r=3$ ) and the corresponding forecast.

As we do not expect periodic components in the forecasts, we can take $h_{1} \neq h_{2}$. We have selected $h_{1}=96$ and $h_{2}=$ 100. The domain of values for $r$ is $[1,3]$ (taking large values of $r$ does not make much sense in this example as we are only interested in forecasting the trend). The total sample size of the samples $F_{t}(t=230, \ldots, 300)$ is therefore $M=$ $(105-96+1)(50-20+1) 3=930$.

In Figure 2 we depict plots of the means $\bar{f}_{t}$, the standard deviations $s_{t}$ and the ranges $R_{t}$ for $t \in[230,300]$ as well as the box-plots of the samples $F_{t}$ for $t=230,240, \ldots, 300$. 


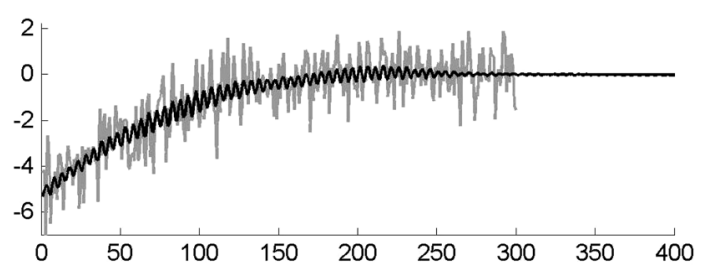

Figure 1. Example 1. The time series (gray), the SSA approximation and the forecast for $L=50$ and $r=3$ (black).

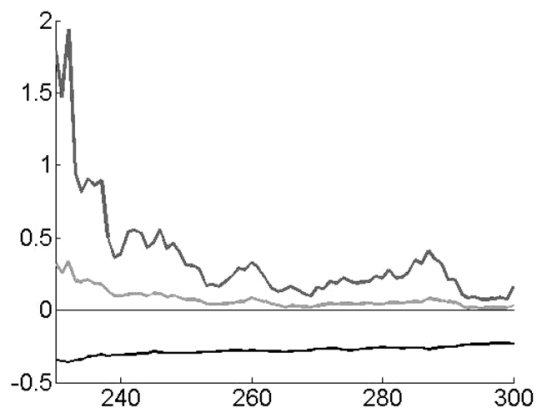

Figure 2. Example 1. Averages $\bar{f}_{t}$ (black), standard deviations $s_{t}$ (light grey), ranges $R_{t}$ (dark grey).

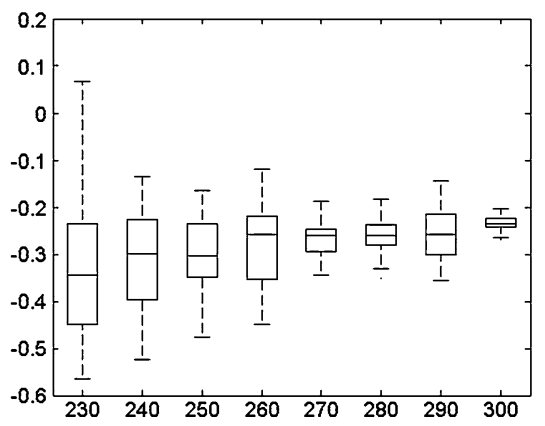

Figure 3. Example 1. Box-plots of the samples $F_{t}$.

Simulated example 2

Let us now consider the time series

$$
x_{t}=t / 200-\left(1-0.2 \cos \left(\frac{\pi t}{75}\right)\right) \cos \left(\frac{\pi t}{5}\right)+\varepsilon_{t}
$$

where $\varepsilon_{t}$ are independent Gaussian random variables with mean 0 and variance $1 / 4$. We only consider the realization of this series shown in Figure 4; this figure also shows a typical SSA approximation (which we obtain for $L=50$ and $r=5$ ) and the corresponding forecast.

There is some seasonality (periodic components) in the series and therefore in the forecasts. We therefore have to take $h_{1} \simeq h_{2}$. We have selected $h_{1}=h_{2}=100$. The values for $r$ are 5 and 7 (taking small values of $r$ does not make much sense as we would miss the periodics; larger values of $r$ would simply lead to the inclusion of noise into the

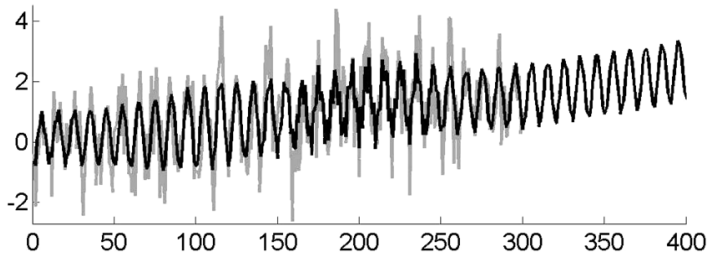

Figure 4. Example 2. The time series (gray), the SSA approximation and the forecast for $L=50$ and $r=5$ (black).

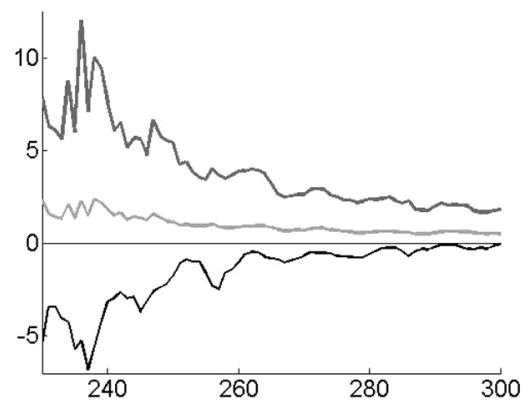

Figure 5. Example 2. Shifted averages $0.9-\bar{f}_{t}$ (black), standard deviations $s_{t}$ (light grey), ranges $R_{t}$ (dark grey).

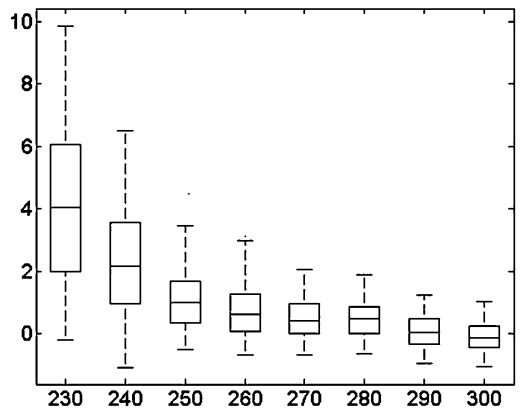

Figure 6. Example 2. Box-plots of the samples $F_{t}$.

approximated series). The total sample size of the samples $F_{t}(t=201, \ldots, 300)$ is therefore $M=1(50-20+1) 2=62$.

In Figure 5 we depict plots of the deviations between the true value $0.9=\mathbf{E} x_{400}$ and the averages $\bar{f}_{t}$, the standard deviations $s_{t}$ and the ranges $R_{t}$ for $t \in[230,300]$ as well as the box-plots of the samples $F_{t}$ for $t=230,240, \ldots, 300$.

\section{ANALYSIS OF EARTH TEMPERATURE RECORDS}

In this section, we apply the methodology described above to analyze the stability of forecasts of Earth temperature records. All the time series are taken from the web-site http://vortex.nsstc.uah.edu/ (National Space Science and Technology Center, USA, NASA). These series represent the temperature on Earth during the last 30 years and are widely discussed in literature, see for example, [4]. 
The series are the so-called temperature anomalies rather than the absolute temperatures (temperature anomalies are computed relative to the base period 1951-1980). Working with anomalies rather than with absolute temperature records is customary in climatology, see for example the publications and web-sites of the Goddard Institute for Space Studies. The first data point in each series is December 1978, the last is December 2009 so that altogether we have $T=373$ data points. The first time moment we start the forecasts is January 2005 implying $T_{0}=314$. We forecast the series until 2018 (longer-term forecasts are very similar) by setting $h_{1}=97, h_{2}=99$.

As in examples above we select the domain $L \in[20,50]$ for the SSA window length $L$. Similar to Example 2, we choose the first $r \in[5,7]$ eigenvectors. Despite a better forecast (with better stability) can be obtained if we optimize the domains of parameters $L$ and $r$ for each individual series, we have fixed the domains to show the robustness of results. Furthermore, the results of our study are very stable with respect to these domains.

We have selected the global temperature on Earth, Northern Hemisphere temperature and North Pole temperature. These three series of temperatures are discussed most often. We have done similar analysis for some other series; the results are presented at the web-site [1]. This web-site also contains more results for the series considered here.

For each of the three chosen temperature series we plot the following.

(i) Figures 7, 10 and 13: the series itself, the SSA approximation and SSA forecast for $L=50$ and $r=7$ computed at the last point $t=T$ (December 2009).

(ii) Figures 8, 11 and 14 (left): the series $-\bar{f}_{t}$, standard deviations $s_{t}$ (light grey), ranges $R_{t}$ (dark grey) for $t=$ $314, \ldots, 373$ (the averages $\bar{f}_{t}$ are always plotted with the minus sign for the purpose of clarity of display).

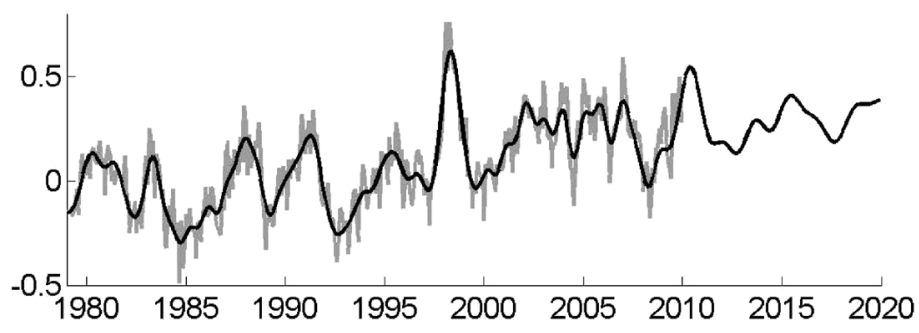

Figure 7. Earth temperature. The time series (gray), the SSA approximation and the forecast for $L=50$ and $r=7$ (black).
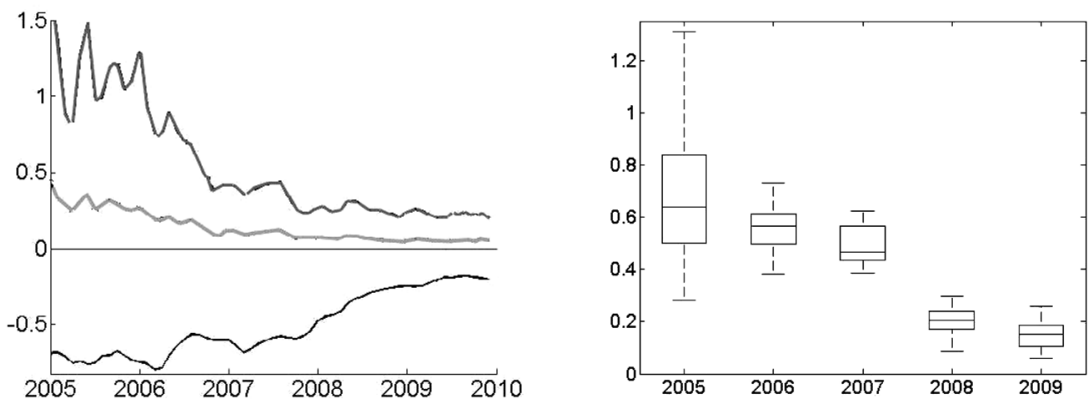

Figure 8. Earth temperature. Left: averages $-\bar{f}_{t}$ (black), standard deviations $s_{t}$ (light grey), ranges $R_{t}$ (dark grey). Right: box-plots of the samples $F_{t}$.
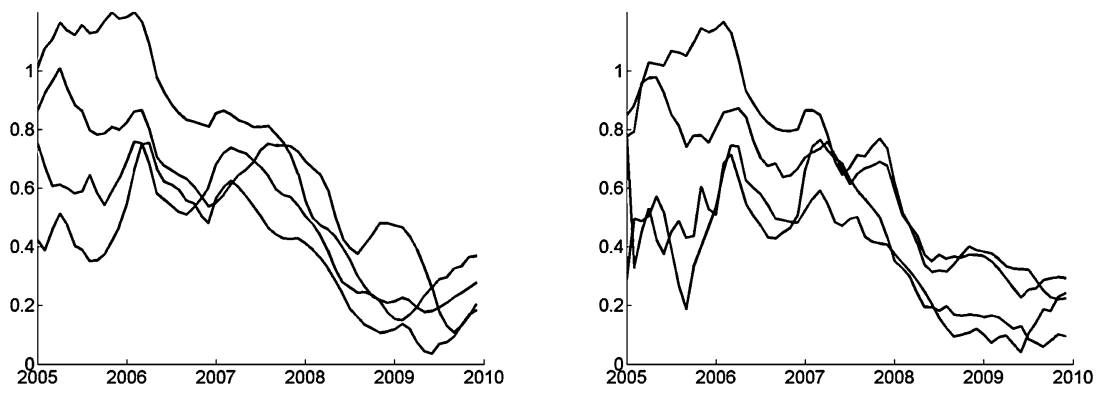

Figure 9. Forecasts for the Earth temperature at Jan 2018; $L=20,30,40,50$ and $r=5$ (left) and $r=7$ (right). 


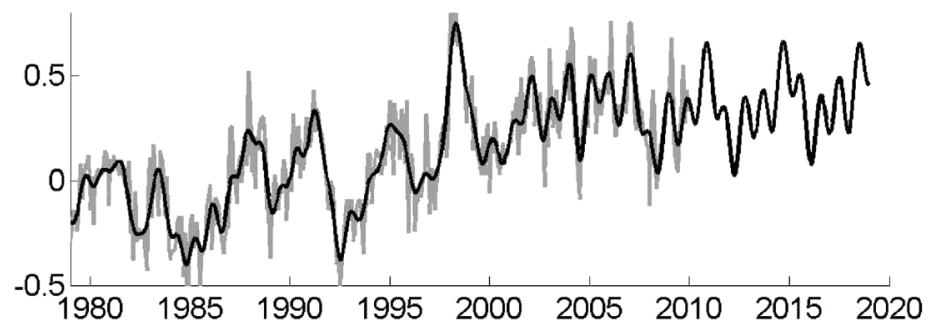

Figure 10. Northern Hemisphere temperature. The time series (gray), the SSA approximation and the forecast for $L=50$ and $r=7$ (black).
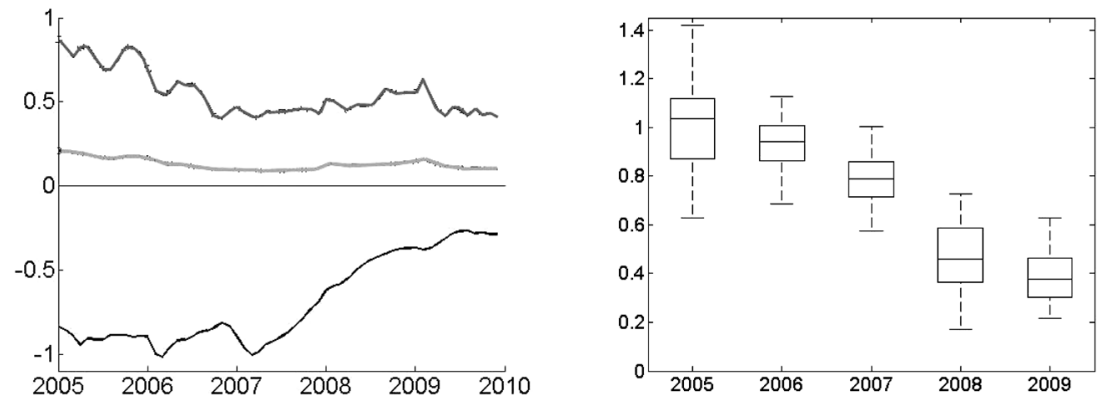

Figure 11. Northern Hemisphere temperature: Left: averages $-\bar{f}_{t}$ (black), standard deviations $s_{t}$ (light grey), ranges $R_{t}$ (dark grey). Right: box-plots of the samples $F_{t}$.
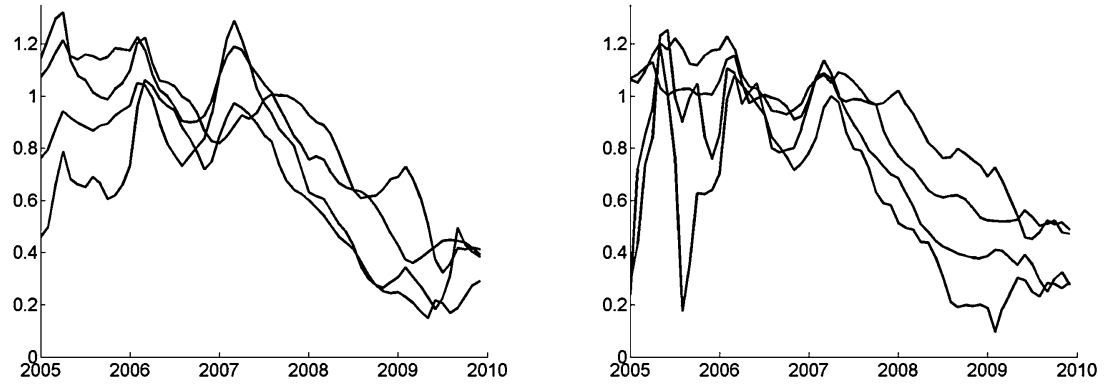

Figure 12. Forecasts for the Northern Hemisphere temperature at Jan 2018; $L=20,30,40,50$ and $r=5$ (left) and $r=7$ (right).

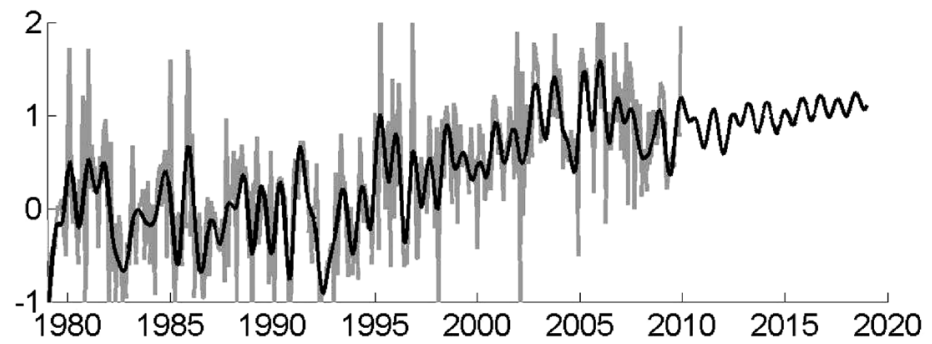

Figure 13. North Pole temperature. The time series (gray), the SSA approximation and the forecast for $L=50$ and $r=7$ (black). 

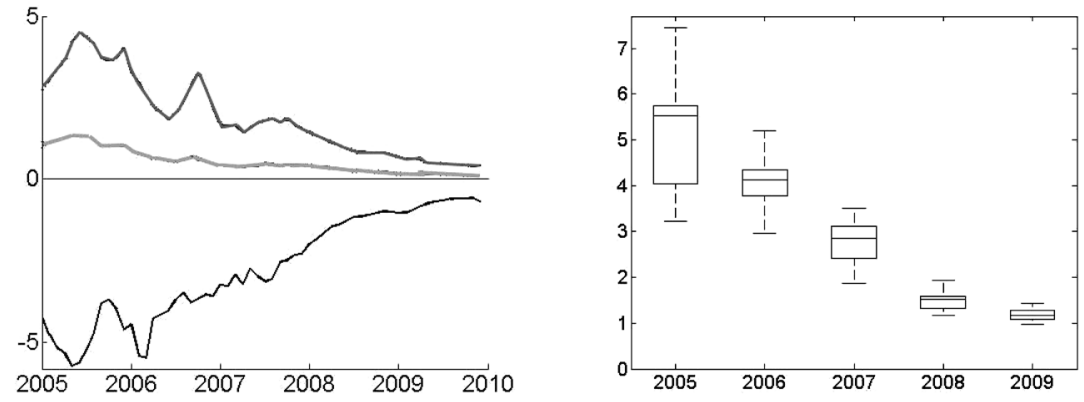

Figure 14. North Pole temperature. Left: averages $-\bar{f}_{t}$ (black), standard deviations $s_{t}$ (light grey), ranges $R_{t}$ (dark grey). Right: box-plots of the samples $F_{t}$.
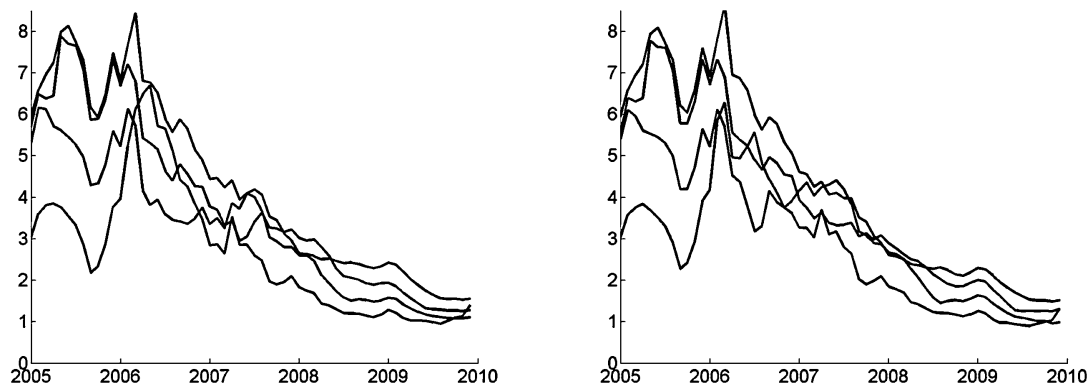

Figure 15. Forecasts for the North Pole temperature at Jan 2018; $L=20,30,40,50$ and $r=5$ (left) and $r=7$ (right).

(iii) Figures 8, 11 and 14 (right): box-plots of the samples $F_{t}$ for $t=325,337,349,361,373$.

(iv) Figures 9, 12 and 15: forecasts for the temperature at January 2018 using the series $x_{1}, \ldots, x_{t}$ for $L=$ $20,30,40,50, r=5,7$ and all $t=314, \ldots, 373$.

Note that the markers on the $\mathrm{x}$-axis in all plots correspond to Januaries. To compare the forecasted values of the temperatures with the recent values, note the average values of these temperatures during the last ten years 2000-2009: 0.222 for Earth; 0.312 for Northern Hemisphere; 0.859 for North Pole.

We can observe that the forecasts made for the series truncated at 2009 and 2010 (or not truncated at all) are much more stable than the forecasts based on series truncated at 2006 and earlier. This can be clearly seen in many more detail in the animated figures presented at the website [1].

We can also observe that the forecasts of the temperature for the next several years are very close to the averaged temperatures during the last ten years 2000-2009. At the same time, the forecasts based on the series truncated at 2005-2006 are significantly larger than these averaged temperatures.

\section{REFERENCES}

[1] Supplementary materials http://earth-temperature.com/stability/

[2] Zhigljavsky, A. (2010). Singular Spectrum Analysis for time series: Introduction to this special issue. Statistics and Its Interface 3, 255-258.

[3] Golyandina, N., Nekrutkin, V. And Zhigluavsky, A. (2001). Analysis of Time Series Structure: SSA and related techniques. Chapman \& Hall/CRC. MR1823012

[4] Plimer I. (2009). Heaven and Earth: Global Warming - The Missing Science. Quartet books, London.

[5] Vautard, R., Yiou, P. and Ghil, M. (1992). Singular-spectrum analysis: A toolkit for short, noisy chaotic signal. Physica D 58, $95-126$.

Andrey Pepelyshev

School of Mathematics and Statistics

Sheffield University

Hounsfield Road

Sheffield, S3 7RH, UK

E-mail address: a.pepelyshev@sheffield.ac.uk

Anatoly Zhigljavsky

School of Mathematics

Cardiff University

Senghennydd Road

Cardiff, CF24 4AG, UK

E-mail address: zhigljavskyaa@cardiff.ac.uk

Received 15 April 2010 